\title{
Estimating the impact of the QCD dynamics on the determination of the high energy astrophysical neutrino flux
}

\author{
Victor P. Gonçalves ${ }^{1, \mathrm{a}}$, Diego R. Gratieri ${ }^{2, \mathrm{~b}}$, Alex S. C. Quadros ${ }^{1, \mathrm{c}}$ \\ ${ }^{1}$ Instituto de Física e Matemática, Universidade Federal de Pelotas (UFPel), Caixa Postal 354, Pelotas, RS CEP 96010-090, Brazil \\ ${ }^{2}$ Escola de Engenharia Industrial Metalúrgica de Volta Redonda, Universidade Federal Fluminense (UFF), Volta Redonda, RJ CEP 27255-125, \\ Brazil
}

Received: 26 November 2020 / Accepted: 20 May 2021 / Published online: 4 June 2021

(C) The Author(s) 2021

\begin{abstract}
The number of ultra-high energy neutrinos arriving at IceCube depends on the energy dependence of the astrophysical neutrino flux and neutrino cross-section. In this paper, we investigate the impact of different assumptions for the description of the QCD dynamics at high energies on the determination of the normalization $\Phi_{\text {Astro }}$ and spectral index $\gamma$ of the astrophysical neutrino flux. The distribution of neutrino events at the IceCube is estimated considering the DGLAP, BFKL, CGC and BBMT approaches and the best estimates for $\Phi_{\text {Astro }}$ and $\gamma$ are determined using a maximum likelihood fit comparing the predictions with the distribution of observed events at IceCube. Moreover, we also investigate if the increase in the effective exposure time expected in IceCube-Gen2 will to allow us to disentangle the QCD dynamical effects from the description of the astrophysical neutrino flux.
\end{abstract}

\section{Introduction}

The detection of ultra-high energies (UHE) neutrinos by the IceCube Neutrino Observatory started a new era in the neutrino physics. The observation of neutrino events with deposited energies in the range between $\mathrm{TeV}$ and $\mathrm{PeV}$ [1-3] has motivated a lot of studies about the production, composition, propagation, and detection of these neutrinos (For a review see, e.g. Refs. [4,5]). In particular, several authors have discussed the current theoretical uncertainty on the main ingredients needed to estimate the number of events in IceCube, which is given

\footnotetext{
a e-mail: barros@ufpel.edu.br (corresponding author)

b e-mail: drgratieri@id.uff.br

ce-mail: alexscq@gmail.com
}

by

$$
\begin{aligned}
d N_{\text {events }}= & T \sum_{\nu+\bar{v}} N_{\text {eff, } \alpha}\left(E_{v}\right) \\
& \times \Phi_{v_{\alpha}}\left(E_{v}\right) \times \sigma_{v_{\alpha}}\left(E_{v}\right) \times d\left(E_{v i s}\right) \times S_{\alpha}\left(E_{v}\right) \times d \Omega,
\end{aligned}
$$

where $T$ is the time of data taken, $N_{e f f, \alpha}\left(E_{v}\right)$ is the effective number of scattering targets, $\Phi_{\nu_{\alpha}}$ is the astrophysical neutrino flux for a neutrino of flavor $\alpha, \sigma_{v_{\alpha}}\left(E_{v}\right)$ is the neutrino-target cross section for a given neutrino energy $E_{v}$ and $S_{\alpha}\left(E_{v}\right)$ is the absorption function, which takes into account the effects of the neutrino flux attenuation inside the Earth. At high energies, the predictions are strongly dependent on the neutrino flux and the neutrino-hadron cross section. One shortcoming present in Eq. (1) is that the event rates constrain only the product of neutrino flux and neutrino-target cross section. Such aspect has motivated the proposition of different strategies to disentangle the physics associated with the neutrinotarget interactions from the properties of astrophysical sources that determine the neutrino flux (see e.g. Refs. [6$8])$.

In recent years, several authors have discussed the treatment of neutrino-hadron cross section $\left(\sigma_{v h}\right)$ at high energies, which is expected to be sensitive to the description of Quantum Chromodynamics (QCD) in the kinematic region of very small values of Bjorken- $x$ and large virtualities $Q^{2}$, not explored by the HERA measurements [9]. The standard framework to calculate the neutral and charged current cross sections is the collinear DGLAP factorization, which predicts that $\sigma_{v h}$ increases with the neutrino energy due to the increasing of the quark and gluon densities inside of hadrons at small- $x$ (see e.g. Ref. [10]). However, new dynamical effects can be present in the unexplored kinematical range probed by the neutrino telescopes, for example those asso- 
ciated with the BFKL dynamics $[11,12]$ or to the non-linear (saturation) corrections [13-15]. In Ref. [16], the authors have investigated the impact on the neutrino-hadron cross sections of the small- $x$ (BFKL) resummation corrections to the DIS coefficient functions and the DGLAP splitting functions and found that its predictions for $\sigma_{v h}$ in the IceCube energy range differ from those derived using the DGLAP formalism, with the difference increasing at larger energies. On the other hand, the fact that the growth of the parton distributions predicted by the DGLAP and BFKL equations is expected to saturate, forming a Color Glass Condensate (CGC) [17-24], has motivated a series of studies about the impact of these effects on $\sigma_{v h}$ in the last decade. The basic idea is that for large energies one expects the transition of the regime described by the linear DGLAP and BFKL dynamics [25-27], where only parton emissions are considered, to a new regime where the physical process of recombination of partons becomes important in the parton cascade and the evolution is given by a non-linear evolution equation [17,18,21-24]. The results from Refs. [28-32] indicate that the saturation effects are non-negligible in the kinematical range probed by the current and future neutrino telescopes. Moreover, we will also consider the approach proposed in Ref. [33] and updated in Refs. [34-36], denoted BBMT hereafter, which is based on the assumption that the proton structure function saturates the Froissart bound at high energies. Such approach takes into account the unitarity corrections at all orders in the strong hadronic interactions. As all these approaches (DGLAP/BFKL/CGC/BBMT), based on distinct assumptions, describe the precise HERA data, the predictions for the behaviour of $\sigma_{v h}$ at very large energies are model dependent.

Our goal in this study is twofold. First, estimate the impact of the distinct treatments of the QCD dynamics, present in the calculation of $\sigma_{v h}$, on the determination of the neutrino flux $\Phi_{v_{\alpha}}$ using the current IceCube data for the energy distribution of events. Second, investigate if the increase in the effective exposure time expected in IceCube-Gen2 [37] will allow us to disentangle the QCD dynamical effects from the description of the astrophysical neutrino flux. This paper is organized as follows. In the next Section, we will present a brief review of the formalism needed to describe the neutrinotarget cross section and the absorption function. Moreover, we will present our assumptions about the neutrino flux, as well as, the approximations assumed in the calculation of the number of events observed by IceCube. In Sect. 3, we will present our predictions for $\sigma_{v h}$ and $S_{\alpha}\left(E_{v}\right)$ considering different descriptions of the QCD dynamics. In addition, predictions from these distinct models are compared with the IceCube data and the associated results for the neutrino flux are presented. Finally, in Sect. 4, we will summarize our main results and conclusions.

\section{Formalism}

In this Section, we will present the models used to describe the distinct ingredients needed to estimate the number of high energy neutrino events in IceCube, described by Eq. (1). Initially, let's discuss the neutrino-target cross section $\sigma_{v_{\alpha}}$. In our calculations, we will consider that the neutrino interaction with the detector can happen through eleven possible neutrino interaction channels, which refers to charged current (CC), neutral current (NC), and resonant scattering due to the Glashow resonance. Differently from the cross sections for the Glashow resonance channels, which are well known, the description of the deep inelastic neutrino scattering depends on the modelling of the QCD dynamics. In what follows, we will describe in more details the charged current (CC) interactions, which proceed through $W$ exchange, but similar expressions can be derived for neutral current (NC) interactions. One has that the total neutrino-hadron cross section for CC interactions is given by [38]

$\sigma_{v h}^{C C}\left(E_{v}\right)=\int_{Q_{\min }^{2}}^{s} d Q^{2} \int_{Q^{2} / s}^{1} d x \frac{1}{x s} \frac{\partial^{2} \sigma^{C C}}{\partial x \partial y}$,

where $s=2 M E_{v}$ with $M$ the hadron mass, $y=Q^{2} /(x s)$ and $Q_{\min }^{2}\left(=1 \mathrm{GeV}^{2}\right)$ is the minimum value of $Q^{2}$ which is introduced in order to stay in the deep inelastic region. Moreover, the differential cross section is given by [38]

$$
\begin{aligned}
\frac{\partial^{2} \sigma_{v h}^{C C}}{\partial x \partial y}= & \frac{G_{F}^{2} M E_{v}}{\pi}\left(\frac{M_{W}^{2}}{M_{W}^{2}+Q^{2}}\right)^{2} \\
& \times\left[\frac{1+(1-y)^{2}}{2} F_{2, C C}^{h}\left(x, Q^{2}\right)-\frac{y^{2}}{2} F_{L, C C}^{h}\left(x, Q^{2}\right)\right. \\
& \left.+y\left(1-\frac{y}{2}\right) x F_{3, C C}^{h}\left(x, Q^{2}\right)\right],
\end{aligned}
$$

where $G_{F}$ is the Fermi constant and $M_{W}$ denotes the mass of the charged gauge boson. As demonstrated e.g. in Ref. [16], the calculation of $\sigma_{v h}$ involves integrations over $x$ and $Q^{2}$, with the integrals being dominated by the interaction with small-x partons and by $Q^{2}$ values of the order of the electroweak boson mass squared.

The treatment of the structure functions $F_{2}, F_{L}$ and $F_{3}$ depends on the framework assumed to describe the QCD dynamics. In the DGLAP formalism, we can apply the collinear factorization theorem and factorise these structure functions as follows

$$
\begin{aligned}
F_{i}\left(x, Q^{2}\right)= & \sum_{a=g, q} \int_{x}^{1} \frac{d z}{z} \\
& \times C_{i, a} \times\left(\frac{x}{z}, Q^{2}\right) f_{a}\left(x, Q^{2}\right) \quad i=2,3, L
\end{aligned}
$$

where $f_{a}$ are the parton distributions of the target, which are assumed universal, and the functions $C_{i, a}$ are the coef- 
ficient functions, which can be computed using perturbation theory and are process-dependent. In Ref. [10], which is one of the benchmark calculations of the neutrino-nucleon cross section, the coefficient functions and the PDFs were estimated using the next-to-leading-order (NLO) DGLAP formalism. On the other hand, in Ref. [16], the authors have estimated the structure functions using the framework of collinear factorization at NNLO, taking into account the small- $x$ BFKL resummation up to next-to-leading logarithmic (NNLx) accuracy. The basic motivation of this approach is to include the BFKL corrections, associated with $\alpha_{s} \ln (1 / x)$ terms that are expected to contribute in the kinematical range probed in neutrino telescopes, in the calculations of the neutrino-hadron cross sections. One has that both approaches predict the increasing of $\sigma_{v h}$ with the neutrino energy, which is expected since they are based on linear evolution equations, which only consider parton emissions $(g \rightarrow g g$ ) and disregard possible recombination effects $(g g \rightarrow g)$ that can contribute in the regime of high partonic density.

The impact of the non-linear (saturation) effects in the neutrino-hadron cross section can be estimated using the color dipole approach and the CGC formalism. In the color dipole approach [39], the structure functions can be factorized in terms of the gauge boson wave function, $\Psi^{G}$, which described the fluctuation of the virtual gauge boson into a $q \bar{q}$ dipole, and the dipole-hadron cross section, $\sigma^{d h}$, that describes the interaction of the color dipole with the target. For charged current interactions, one has that the $F_{2}^{C C}$ structure function is expressed in terms of the transverse and longitudinal structure functions, $F_{2}^{C C}=F_{T}^{C C}+F_{L}^{C C}$ which are given by

$$
\begin{aligned}
F_{T, L}^{C C}\left(x, Q^{2}\right)= & \frac{Q^{2}}{4 \pi^{2}} \int_{0}^{1} d z \int d^{2} \boldsymbol{r} \mid \Psi_{T, L}^{W} \\
& \times\left.\left(\boldsymbol{r}, z, Q^{2}\right)\right|^{2} \sigma^{d h}(\boldsymbol{r}, x)
\end{aligned}
$$

where $r$ denotes the transverse size of the dipole, $z$ is the longitudinal momentum fraction carried by a quark and $\Psi_{T, L}^{W}$ are the wave functions of the virtual charged gauge boson associated with their transverse or longitudinal polarizations. In the CGC formalism [17, 18,21-24], the dipole-target cross section can be computed in the eikonal approximation, being given by

$\sigma^{d h}(x, \boldsymbol{r})=2 \int d^{2} \boldsymbol{b} \mathcal{N}^{h}(x, \boldsymbol{r}, \boldsymbol{b})$,

where $\mathcal{N}^{h}$ is the forward dipole-target scattering amplitude for a given impact parameter $\boldsymbol{b}$ which encodes all the information about the hadronic scattering, and thus about the non-linear and quantum effects in the hadron wave function. The Balitsky-JIMWLK hierarchy [17, 18,21-24,40-42] describes the energy evolution of the dipole-target scattering amplitude, which decouples in the mean-field approximation and boils down to the Balitsky-Kovchegov (BK) equation [40-42]. During the last decades, several phenomenological models based on the Color Glass Condensate formalism [1315] have been proposed to describe the HERA data taking into account the non-linear effects in the QCD dynamics. In general, such models differ in the treatment of the impact parameter dependence and/or of the linear and non-linear regimes. In our analysis, we will consider the IIMS model proposed in Ref. [43] and updated in Ref. [44], which is based on the asymptotic solutions of the BK equation and that describe with success the high precision HERA data, as shown in Ref. [45]. In this model, the dipole-proton scattering amplitude is given by

$\mathcal{N}^{p}(x, \boldsymbol{r}, \boldsymbol{b})=S(\boldsymbol{b}) \times \begin{cases}\mathcal{N}_{0}\left(\frac{r Q_{s}}{2}\right)^{2\left(\gamma_{s}+\frac{\ln \left(2 / r Q_{s}\right)}{\kappa \lambda y}\right)} & r Q_{s} \leq 2(7) \\ 1-e^{-A \ln ^{2}\left(B r Q_{s}\right)} & r Q_{s}>2,\end{cases}$

where $S(\boldsymbol{b})$ is the proton profile function, $\chi$ is the LO BFKL characteristic function, $\kappa=\chi^{\prime \prime}\left(\gamma_{s}\right) / \chi^{\prime}\left(\gamma_{s}\right)$ and $y=\ln (1 / x)$. Moreover, the coefficients $A$ and $B$ are determined uniquely from the condition that $\mathcal{N}^{p}$ and its derivative with respect to $r Q_{s}$ are continuous at $r Q_{s}=2$. Finally, in Refs. [3336], the authors proposed an alternative way to take into account of the unitarity (saturation) effects at all orders motivated by the successful descriptions of hadron-hadron and photon-hadron total cross sections over many orders of magnitude obtained with the same saturated Froissart functional form, i.e., $\sigma_{i h} \propto \ln ^{2} s(i=\gamma, p)$. The main assumption in the BBMT approach for neutrino-hadron interactions is that growth on the proton structure function is limited by the Froissart bound at high hadronic energies, giving a $\ln ^{2}(1 / x)$ bound on $F_{2}$ as Bjorken $x \rightarrow 0$, which implies an exact bound of $\ln ^{3} E_{v}$ for the $v N$ scattering. As demonstrated in Ref. [35], such approach is able to describe the combined HERA data.

Another important ingredient in the calculation of the number of high energy neutrino events in IceCube is the absorption function for the neutrinos while it crosses the Earth, defined by

$S^{j}\left(E_{v}\right)=\int_{-1}^{0} d \cos \left(\theta_{z}\right) P_{\text {shad }}^{j}\left(E_{v}\right)$,

where $\theta_{z}$ is the zenith angle and $P_{\text {shad }}^{j}\left(E_{v}\right)$ is the the probability of neutrino interaction while cross the Earth, which can be expressed as follows [46]

$P_{\text {Shad }}^{j}\left(E_{\nu}\right)=\exp \left\{-\frac{z_{j}\left(\theta_{z}\right)}{\mathcal{L}_{\text {int }}^{j}}\right\}$.

One has that $j$ represents a nucleon $N$ or an electron $e, z_{j}\left(\theta_{z}\right)$ is the amount of matter that neutrinos feel while travel across 
the Earth and the interaction lengths for scattering with nucleons and electrons are given respectively by

$$
\begin{aligned}
\mathcal{L}_{i n t}^{N} & =\frac{1}{N_{A} \sigma_{v N}\left(E_{v}\right)} \\
\mathcal{L}_{i n t}^{e} & =\frac{1}{\langle Z / A\rangle N_{A} \sigma_{\bar{v}_{e} e}\left(E_{v}\right)},
\end{aligned}
$$

where $N_{A}$ is the Avogadro's number, $Z(A)$ is the atomic (mass) number factor and the factor $\langle Z / A\rangle$ is the average ratio between electrons $(Z=e=p)$ and nucleons $(A=p+n)$. Moreover, one has that $z_{j}\left(\theta_{z}\right)$ is given by

$z_{j}\left(\theta_{z}\right)=\int_{0}^{r\left(\theta_{z}\right)} \rho_{j}(r) d r$

where $r\left(\theta_{z}\right)=-2 R_{\text {Earth }} \cos \theta_{z}$ is the total distance travelled by neutrinos, $\rho_{j}(r)\left[\mathrm{g} \mathrm{cm}^{-3}\right]$ is the density profile of the Earth. In Ref. [30], we demonstrated that $P_{\text {shad }}^{j}\left(E_{v}\right)$ can be written as follows

$P_{\text {Shad }}^{j}\left(E_{\nu}\right)=\exp \left\{-\kappa_{j} \sigma_{\nu j}\left(E_{\nu}\right) \int_{0}^{r\left(\theta_{z}\right)} \rho_{j}(r) d r\right\}$,

where $\kappa_{N}=N_{A}$ and $\kappa_{e}=\langle Z / A\rangle \cdot N_{A}$. This result demonstrates that $P_{\text {Shad }}^{j}\left(E_{v}\right)$ is strongly sensitive to the description of the neutrino-target cross section and the amount of matter crossed by the neutrinos. As shown in Ref. [30], such dependence is also present in the absorption function $S^{j}\left(E_{v}\right)$. In this paper, we will estimate $P_{\text {shad }}^{j}\left(E_{v}\right)$ and $S^{j}\left(E_{v}\right)$ considering different models for the (anti) neutrino-nucleon cross section and, for comparison, we also present the results for (anti) neutrino-lepton interactions.

One has that in order to compute the number of interactions in IceCube detector we must integrate the product of astrophysical neutrino fluxes with the respective neutrino interaction cross sections, absorption functions and effective number of scattering targets. As IceCube does not distinguish charge, we have summed over the neutrino and antineutrino contributions. In our analysis, we will assume $T=2078$ days and that the effective number of scattering targets is given by

$$
N_{e f f}\left(E_{v}\right)=N_{A} \times V_{e f f}\left(E_{v}\right)=N_{A} \times M_{e f f}\left(E_{v}\right) / \rho_{e f f} .
$$

where the effective detector masses, $M_{e f f, \alpha}$, are given in [47]. The last ingredient that we must to specify is the astrophysical neutrino flux. One has that its origin is still a theme of intense debate. However, the experimental data is so far consistent with results expected from extra-galactic sources, presenting isotropy and no correlation with the galactic plane. As usual in the literature, we will assume the same astrophysical neutrino flux for the three neutrino flavors, $\Phi_{v_{e}}=\Phi_{v_{\mu}}=\Phi_{v_{\tau}}=\Phi_{0}$, which from [48] is given by

$\Phi_{v}\left(E_{v}\right)=\frac{\Phi_{\text {astro }}}{\left(E_{v} / 100 \mathrm{TeV}\right)^{\gamma}}($ f.u. $)$,

where $\gamma$ is the power law index and $\Phi_{\text {Astro }}=3 \Phi_{0}$ defines the normalization and the flux unit is defined as f.u. $\equiv$ $10^{-18} \mathrm{GeV}^{-1} \mathrm{~s}^{-1} \mathrm{sr}^{-1} \mathrm{~cm}^{-2}$. In our analysis, we will estimate the distribution of neutrino events at the IceCube assuming different assumptions for the QCD dynamics and we will determine the best estimates for $\Phi_{\text {Astro }}$ and $\gamma$ using a maximum likelihood fit by the comparison of our predictions with the distribution of observed events. Our goal is to verify if the current and future data can be used to remove the degeneracy between the neutrino-target cross section and astrophysical flux present in Eq. (1).

\section{Results}

Initially, let's present in Fig. 1a our predictions for the energy dependence of the neutrino-target cross section. For the CC neutrino-nucleon case, we present the predictions of the distinct approaches for the treatment of the QCD dynamics discussed in the previous Section. In particular, we denote by CGC (IIMS) the results calculated using the latest version [45] of the non-linear approach derived in Refs. [43,44], in which the free parameters were fitted using the high precision HERA data for the proton structure function. For the standard DGLAP calculations, we have estimated the cross section using the CT14 parameterization [49] and the associated results are denoted by DGLAP (CT14) hereafter. On the other hand, the results obtained adding the small- $x$ BFKL contributions in Ref. [16] will be denoted by BFKL (BGR18) in what follows. The uncertainty bands present in the CT14 and BGR18 predictions are also shown. Finally, for the BBMT approach, we consider the results obtained in Ref. [36]. One has that the DGLAP and BFKL results are similar in the IceCube energy range, but its central predictions are slightly distinct for larger energies. The inclusion of the non-linear effects, as predicted by the CGC (IIMS) approach, implies a smaller cross section in the energy range $10^{6} \mathrm{GeV} \leq E_{v} \leq 10^{8} \mathrm{GeV}$. At larger energies, the associated predictions are inside of the uncertainty bands of the CT14 and BGR18 predictions. On the other hand, the BBMT result is similar to the DGLAP and BFKL predictions in the IceCube energy range, but implies a strong reduction of the cross section at larger neutrino energies. The predictions for the antineutrino-electron cross section are also presented in Fig. 1a taking into account the presence of the Glashow resonance which is expected for neutrinos energies of the order of $E_{v, r e s}=\frac{M_{W}^{2}}{2 m_{e}} \approx 6.3 \mathrm{PeV}$. Our results demonstrate that antineutrino-electron scattering becomes equal or greater 


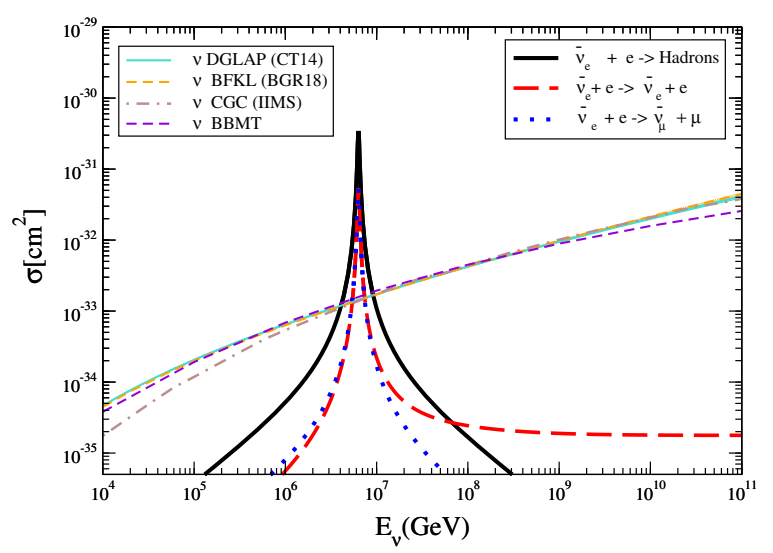

(a)

Fig. 1 a Energy dependence of the neutrino-target cross-sections. b Neutrino absorption by the Earth as a function of neutrino energy. Interactions with nucleon are calculated for the different QCD mod-

than $\mathrm{CC}$ neutrino-nucleon cross-section in the energy range characterized by $10^{6} \mathrm{GeV} \leq E_{v} \leq 2 \times 10^{7} \mathrm{GeV}$. The results presented in Fig. 1a indicate that the predictions for large neutrino energies can differ by a factor $\geq 2$ depending on the approach assumed to treat the QCD dynamics.

In Fig. $1 \mathrm{~b}$ we present our predictions for the absorption function $S\left(E_{v}\right)$. We have that the distinct predictions for $v N$ interactions are very similar for $E_{v} \leq 10^{8} \mathrm{GeV}$, with the difference between the predictions reaching $10 \%$ at $80 \mathrm{TeV}$. On the other hand, at larger energies, we have that the difference between the BFKL (BGR18) and BBMT predictions increases and becomes a factor 2 at $E_{v} \approx 10^{10} \mathrm{GeV}$, with the BBMT one being an upper bound. From Eq. (1), one must expect that the higher is the neutrino-nucleon crosssection, the higher is the total number of events. However, a higher cross-section also implies higher neutrino absorption inside the Earth and, consequently, a smaller number of events in the detector for the upward direction. Another important aspect is that as the neutrino flux decreases with the energy with a power like behaviour, the number of expected events at IceCube and/or future observatories should be small for these energies. Consequently, the difference of a factor two between the predictions has a strong impact on the analysis and interpretation of the possible few events that should be observed.

As discussed above, in order to compute the number of interactions in IceCube detector we must integrate the product of such fluxes given in Eq. (14) with the respective neutrino interaction cross-sections and effective detector masses, $M_{e f f, \alpha}$. The free parameters in our analysis are the normalization $\Phi_{\text {Astro }}$ and the power-law index $\gamma$ of the astrophysical neutrino flux, which will be determined by the comparison of our predictions with the IceCube data. Notice that the quantities in Eq. (1) are expressed in terms of neutrino

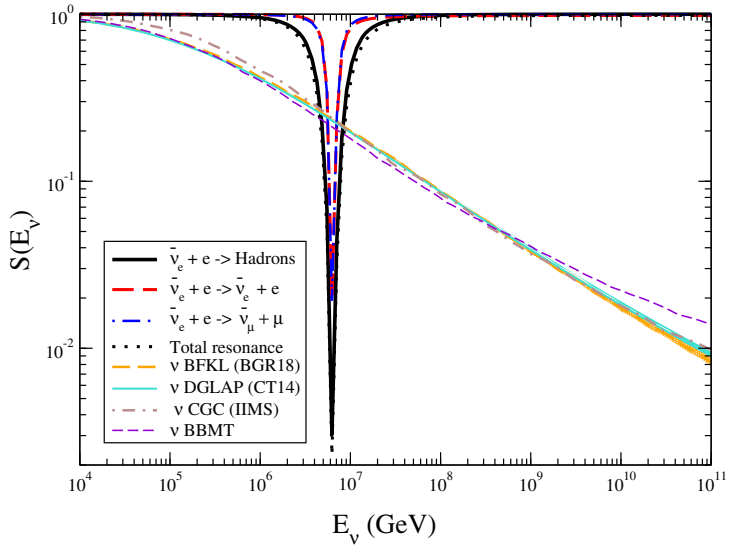

(b)

els. Anti electron-neutrino interaction with electrons in the medium due to Glashow resonance are also taken into account

energy, while IceCube data are given in terms of the amount of electromagnetic energy deposited in the detector. Therefore, we assume the relations between these two quantities for each kind of neutrino interaction as they are given in [50]. Moreover, to include the neutrino events due to the Glashow Resonant interaction with the detector, in our analysis we assume that the branching ratio for the production of the final states $\left(e^{-}, \mu^{-}\right.$, hadron Shower) are respectively $15 \%, 15 \%, 70 \%$ [51]. Also, we assume that in the resonant neutrino scattering, the produced hadronic shower carries all the incoming neutrino energy, with at least $90 \%$ of efficiency in the detection. For the leptonic final states, we assume the same averaged inelasticity as for the C.C. neutrino interaction. Furthermore, in this work we are interested in the distribution of neutrino events in terms of the deposited energy in the interval $60 \mathrm{TeV}<E_{E . M .}<10 \mathrm{PeV}$ and considers all the incoming neutrino directions.

In order to estimate $\Phi_{\text {Astro }}$ and $\gamma$ for the distinct QCD models, we will perform a data analysis of the IceCube UHE neutrino data through the Maximum Likelihood formalism. Given the low statistics, we assume that the data follow the Poisson Distribution. To take into account the systematic errors we use the Pull Method [52]. Moreover, Wilks' Theorem [53] states that the statistic $-2 \ln (\mathcal{L})$ follows a distribution of the $\mathcal{X}^{2}$ type. In this context, one can write the $\mathcal{X}^{2}$ function as

$$
\begin{aligned}
\mathcal{X}^{2} \equiv & -2 \ln (\mathcal{L})+\text { Systematics } \\
= & 2 \sum_{i=1}^{n}\left\{\left(E_{i}-O_{i}\right)+O_{i} \ln \left(\frac{O_{i}}{E_{i}}\right)\right\} \\
& +\sum_{j=1}^{m}\left(\frac{\theta_{j}-\theta_{j}^{*}}{\sigma_{j}}\right)^{2},
\end{aligned}
$$




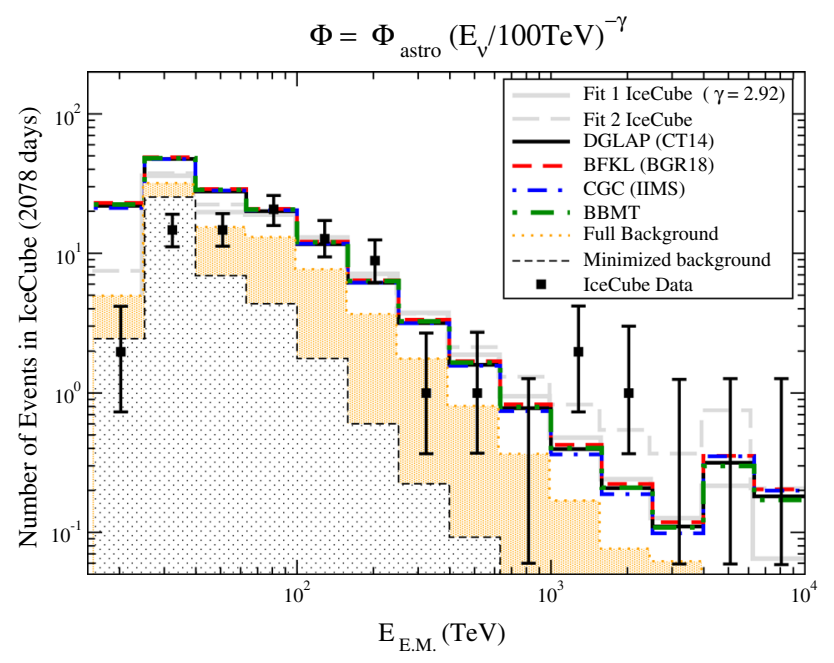

Fig. 2 The number of neutrino events are compared with our results for the energy distribution of events. For comparison, the fits obtained by IceCube Collaboration are also shown (grey lines). Our results (colored lines) are calculated for the respective B.F. P. values for the extragalactic neutrino flux shown in Table 1

where prior information about the values for some parameters $\theta_{j}$ are included in the likelihood function. Gaussian penalties then reject solutions where the best fit value for the parameter $\theta_{j}^{*}$ is different from its prior value. We include the systematic errors relative to the normalization of the different backgrounds: atmospheric muon flux, $\Phi_{\mu}$, conventional atmospheric neutrino flux, $\Phi_{\nu}$, atmospheric prompt neutrinos, $\Phi_{v_{p t}}$. Also, we take into account the error associated with the energy resolution, $\delta E$. The respective priors and allowed intervals are defined for example in [54] and are respectively $(1.00 \pm 0.5 ; 1.00 \pm 0.30 ; 0.00 \pm 0.65 ; 1.00 \pm 0.15)$. For the four QCD models, we found that the values for the pull parameters associated with the best-fit point (B.F.P.) are consistent with each other and with the values reported by [54] in few percent level. The only exception is that in our analysis a slightly higher contribution of prompt neutrinos, $\Phi_{v_{p t}} \approx 0.20$, was preferred in all the cases. In any case, this value is in agreement with the allowed interval of $68 \%$ of confidence level (C.L.) reported in [54] for this parameter. Indeed, we obtained a good description of the energy dependence of IceCube data for all four models. In Table 1 we show the best fit points we obtained for the parameters $\gamma$ and $\Phi_{\text {astro }}$ for the different interaction models we use. As can be seen, the variation in the value of the minimum of $\mathcal{X}^{2}$ is of $\approx 3 \%$.

In Fig. 2 we show our results for the number of events obtained when the respective values of the B.F.P. are assumed for both extra-galactic neutrino flux and neutrino background (colored lines) and compare it with the IceCube data. We also show in the shaded areas the background for the case where all the normalizations are set to one (which includes the limit
Table 1 Best fit values for the extra-galactic neutrino flux derived assuming different approaches for the QCD dynamics

\begin{tabular}{lll}
\hline$\gamma \pm \delta \gamma$ & $\Phi_{\text {astro }} \pm \delta \Phi_{\text {astro }}$ (f.u.) & $\mathcal{X}_{\text {min }}^{2}$ \\
\hline $2.90_{-0.22}^{+0.23}$ & DGLAP (CT14) & \\
& $0.64_{-0.28}^{+0.33}$ & 10.81 \\
$2.94_{-0.26}^{+0.19}$ & BFKL (BGR18) & \\
& $0.72_{-0.20}^{+0.44}$ & 10.90 \\
$3.03_{-0.18}^{+0.20}$ & CGC (IIMS) & \\
$2.94_{-0.25}^{+0.26}$ & $1.04_{-0.47}^{+0.48}$ & 11.02 \\
\hline
\end{tabular}

at $90 \%$ of C.L. of the prompt neutrino background reported in [48]). We call it Full Background. On the other hand, the predictions derived using the values we found for the B.F.P. are denoted by Minimized Background. From Fig. 2 and Table 1 it is clear that the four different QCD dynamics led to an equivalently good description of the energy dependence of the IceCube data set. Moreover, the predictions for the power index B.F.P. due to all the models do agree in the $68 \%$ limit and the relative difference between the $\Delta \mathcal{X}_{\min }^{2}$ from each model is negligible (3\%). Nevertheless, when we compare the B.F.P. due to the DGLAP (CT14) and CGC (IIMS) models, we obtained a difference of a factor $\approx 1.6$ in the value of $\Phi_{\text {astro }}$, while for the power index $\gamma$ the maximum variation we found is of the order of $\approx 5 \%$. Notice that this difference of $5 \%$ has the same order of magnitude as $\delta \gamma$, whose values associated with the different models are contained in the interval $6 \% \leq \delta \gamma \leq 9 \%$, as it can be seen in Table 1 .

In what follows, we show our results for the sensitivity of the energy distribution of IceCube events to the extragalactic neutrino flux parameters. In the central panel of Fig. 3, we show our results for the allowed region for the extra-galactic flux parameters. For comparison, the results from the IceCube Collaboration summarized in [48] are also shown both for the B.F.P. and the allowed region at $68 \%$ of confidence level. In the two auxiliary panels, we show our results for the marginalization with respect to the respective parameters. It is not necessary to say that our goal is not to determine what are the B.F.P. for the extra-galactic neutrino flux but to estimate how these values depend on the assumptions about QCD dynamics at such high neutrino energies. For instance, the significantly smaller cross-section resulting from the CGC (IIMS) model implies that the $\mathcal{X}^{2}$ analysis prefers a higher value of the $\Phi_{\text {astro }}$ parameter when we compare with the values favored when we use the DGLAP (CT14), BFKL (BGR18) and BBMT models. Nonetheless, the expected number of events in the more energetic bins is mostly due to the product of extra-galactic neutrino flux times the Glashow resonant cross-section. Indeed, the higher value 


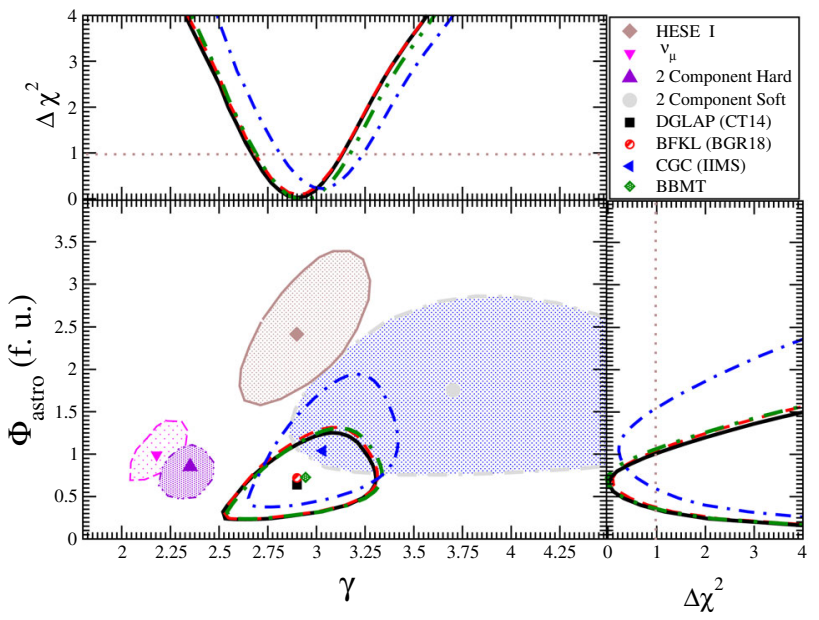

Fig. 3 Likelihood analysis for the High - Energy Starting Events (HESE). Our results are compared with the different analyses performed by the IceCube Collaboration in Ref. [48], which consider both the energy dependence and the angular distribution of events. In all cases points refer to the best fit and the allowed region at $68 \%$ of C.L. is also shown

of the $\Phi_{\text {astro }}$ needed by the CGC (IIMS) model to explain the events in the interval of $60 \mathrm{TeV} \leq E_{v i s} \leq 5 \mathrm{PeV}$, the higher the number of events due to Glashow resonance. Henceforth, despite its small statistical significance, the number of events observed around $6 \mathrm{PeV}$ could theoretically contribute to solving the problem of entanglement between the extra-galactic neutrino flux and C.C. neutrino cross-section. We found that a slightly higher value of $\gamma$ is sufficient to compensate for the higher flux normalization necessary in the CGC (IIMS) analysis, and do not overestimate the number of events due to the Glashow resonance. At the same time, such increment on $\gamma$ is not large enough to worse the description of the other bins.

Finally, the IceCube-Gen2 is the planned upgrade in the IceCube observatory for the next future [37]. As pointed out in [8], the net effect of such improvement is an increment on the effective exposure time, which could be of a factor of 20 (40) in the case of 10 (20) years of IceCube Gen2 data taking. This would lead to a thousands of events in the energy domain we are interested in here. As a consequence, it is expected to allow the discrimination between distinct QCD approaches. To extend the analysis to IceCube Gen2, we assume our predictions for the number of events derived using the DGLAP (CT14) approach, renormalized by the new detector exposure, as being the number of events that will be observed in the future. In this analysis, we fix the pull parameters as being the respective B.F.P. obtained above, and which do agree with the values reported in cite Aartsen: $2015 \mathrm{knd}$. The only exception was the prompt neutrino contribution to the background, which we let vary in the region indicated in the same reference. In Fig. 4 we show our predictions for the different exposures considered. One has for the BFKL (BGR) model that the red lines represent the predictions for the central value of $\mathrm{CC}$ and $\mathrm{NC}$ neutrino cross-sections, and the shaded red areas refer to the results of the superposition of the predictions for the upper and lower limits of these cross-sections. Given the magnitude of the errors in the BFKL (BGR) model, the effect of consider it in the analysis is negligible. The first point to notice is that it is clear that the increase in the detector exposition, and consequently in the number of events, implies the reduction in both the allowed regions associated with the neutrino flux parameters at $68 \%$ and $95 \%$ of C.L. The results presented in Fig. 4a indicate that despite the differences in the B.F.P., there is an overlap of the allowed regions associated with the flux parameters. This superposition at $68 \%$ of C.L. implies in a degree of compatibility between the predictions associated to the BFKL (BGR18), BBMT and CGC (IIMS) models. Indeed, the overlap between the BFKL (BGR18) and BBMT predictions is observed in all the four panels of Fig. 4. On the other hand, the results presented in Fig. $4 \mathrm{~b}$ and $\mathrm{c}$ show that the increasing of the time of data taken in fact reduces the region of overlap between the BFKL (BGR18) and CGC (IIMS) predictions, but they are still compatible at $68 \%$ and $95 \%$ respectively. Finally, Fig. 4d refers to twenty years of exposure combined with the optimistic assumption that the error in the normalization of the prompt neutrino flux is reduced to $15 \%$. For this case, the superposition of the allowed regions for the flux parameters associated to the BFKL (BGR18) and CGC (IIMS) models is no longer present. This is an indication that, in the optimistic case, the increase of the detector exposure combined with the reduction of systematic uncertainties should lead to discrimination at $95 \%$ of C.L. between the associated predictions for the flux parameters. However, it is important to emphasize that even in the optimistic case it is not possible to discard any of the dynamical models considered here. One has that assuming that the predictions due to these different models are valid, then the conclusions about what are the values of the flux parameters $\gamma$ and $\Phi_{\text {astro }}$ can be considerably different in each case. Indeed, the values of the B.F.P. differ for a factor respectively of 1.05 and 1.5. Our results indicate that the uncertainties in the prompt neutrino flux play an important role in the description of the data and further advances in the knowledge about the prompt neutrinos would positively impact on the sensitivity of the IceCube data to the QCD dynamics.

\section{Summary}

The study of the UHE events at the IceCube is expected to improve our understanding about the origin, propagation, and interaction of neutrinos. In particular, the recent data has been used to constrain the energy behavior of the astro- 


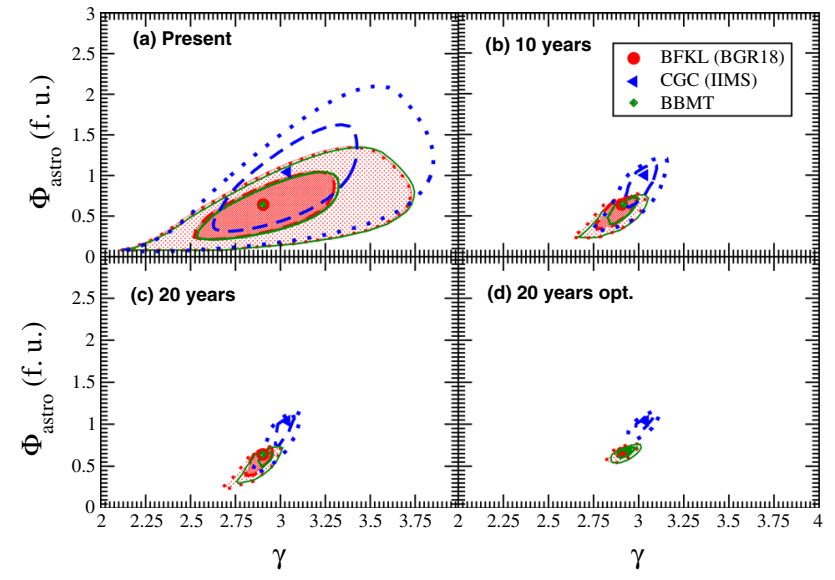

Fig. 4 Effects in the allowed region of parameters due to increments in the IceCube exposition. a The present exposure associated to 2078 days of data taking. b Predictions for ten years of IceCube Gen2. c Predictions for twenty years of IceCube Gen2. d Predictions for twenty years of IceCube Gen 2 derived assuming that the error in the normalization of prompt neutrinos is reduced from the current $65-15 \%$. In all the cases, we assume the DGLAP (CT14) prediction as the observed number of events

physical neutrino flux as well as to constrain the neutrinohadron cross section. One has that variations in these quantities are expected to modify the flux and event rate at IceCube detector. In this paper, we have investigated the impact of different assumptions for the QCD dynamics on the determination of the astrophysical flux. We have estimated the distribution of neutrino events at the IceCube considering the DGLAP, BFKL, CGC and BBMT approaches and find the best estimates for $\Phi_{\text {astro }}$ and $\gamma$ using a maximum likelihood fit comparing the predictions with the distribution of observed events at IceCube. Our results indicated that concerning the data description, the modifications in the normalization and energy dependence of the neutrino-nucleon cross section due to the different dynamical approaches can be compensated by different values for the parameters neutrino flux normalization, $\Phi_{\text {astro }}$, and the power index $\gamma$, in such a way that all the models can describe de data successfully and cannot be disregard even at $68 \%$ of C.L. Moreover, we also investigated if the increase in the effective exposure time expected in IceCube-Gen2 will allow us to disentangle the QCD dynamical effects from the description of the astrophysical neutrino flux. For this case, we have verified that the higher number of events implies the reduction of the overlap of the allowed areas for the neutrino flux parameters. However, our results pointed out that the increase of the detection exposure is not enough to allow us to fully discriminate between the models studied and, consequently, to constrain the description of the QCD dynamics using only the data for the energy dependence of the number of events observed in neutrino telescopes.
Acknowledgements This work was partially financed by the Brazilian funding agencies CNPq, FAPERGS and INCT-FNA (process number 464898/2014-5). Funding was provided by Conselho Nacional de Desenvolvimento Científico e Tecnológico.

Data Availability Statement This manuscript has no associated data or the data will not be deposited. [Authors' comment: This is a theoretical research study, and is based upon analysis of the public experimental data, so no additional data are associated with this work.]

Open Access This article is licensed under a Creative Commons Attribution 4.0 International License, which permits use, sharing, adaptation, distribution and reproduction in any medium or format, as long as you give appropriate credit to the original author(s) and the source, provide a link to the Creative Commons licence, and indicate if changes were made. The images or other third party material in this article are included in the article's Creative Commons licence, unless indicated otherwise in a credit line to the material. If material is not included in the article's Creative Commons licence and your intended use is not permitted by statutory regulation or exceeds the permitted use, you will need to obtain permission directly from the copyright holder. To view a copy of this licence, visit http://creativecomm ons.org/licenses/by/4.0/.

Funded by SCOAP $^{3}$.

\section{References}

1. M.G. Aartsen et al. [IceCube Collaboration], Science 342(6161), 1242856 (2013)

2. M.G. Aartsen et al., IceCube Collaboration. Phys. Rev. Lett. 111 (2013)

3. M.G. Aartsen et al., IceCube. Phys. Rev. Lett. 113 (2014)

4. T. Gaisser, F. Halzen, Ann. Rev. Nucl. Part. Sci. 64(4), 1 (2014)

5. S.R. Klein, arXiv:1906.02221 [astro-ph.HE]

6. E. Borriello, A. Cuoco, G. Mangano, G. Miele, S. Pastor, O. Pisanti, P.D. Serpico, Phys. Rev. D 77 (2008)

7. S. Hussain, D. Marfatia, D.W. McKay, Phys. Rev. D 77 (2008)

8. L.A. Anchordoqui, C.G. Canal, J.F. Soriano, Phys. Rev. D 100(10), 103001 (2019)

9. H. Abramowicz et al., [H1 and ZEUS], Eur. Phys. J. C 75(12), 580 (2015)

10. A. Cooper-Sarkar, P. Mertsch, S. Sarkar, JHEP 08, 042 (2011)

11. E.A. Kuraev, L.N. Lipatov, V.S. Fadin, Sov. Phys. JETP 45, 199204 (1977)

12. I.I. Balitsky, L.N. Lipatov, Sov. J. Nucl. Phys. 28, 822-829 (1978)

13. F. Gelis, E. Iancu, J. Jalilian-Marian, R. Venugopalan, Ann. Rev. Nucl. Part. Sci. 60, 463 (2010)

14. H. Weigert, Prog. Part. Nucl. Phys. 55, 461 (2005)

15. J. Jalilian-Marian, Y.V. Kovchegov, Prog. Part. Nucl. Phys. 56, 104 (2006)

16. V. Bertone, R. Gauld, J. Rojo, JHEP 01, 217 (2019)

17. J. Jalilian-Marian, A. Kovner, L. McLerran, H. Weigert, Phys. Rev. D 55, 5414 (1997)

18. J. Jalilian-Marian, A. Kovner, H. Weigert, Phys. Rev. D 59, 014014 (1999)

19. J. Jalilian-Marian, A. Kovner, H. Weigert, Phys. Rev. D 59, 014015 (1999)

20. J. Jalilian-Marian, A. Kovner, H. Weigert, Phys. Rev. D 59, 034007 (1999)

21. A. Kovner, J.G. Milhano, H. Weigert, Phys. Rev. D 62, 114005 (2000)

22. H. Weigert, Nucl. Phys. A 703, 823 (2002)

23. E. Iancu, A. Leonidov, L. McLerran, Nucl. Phys. A 692, 583 (2001) 
24. E. Ferreiro, E. Iancu, A. Leonidov, L. McLerran, Nucl. Phys. A 701, 489 (2002)

25. V.N. Gribov, L.N. Lipatov, Sov. J. Nucl. Phys. 15, 438 (1972)

26. G. Altarelli, G. Parisi, Nucl. Phys. B 126, 298 (1977)

27. YuL Dokshitzer, Sov. Phys. JETP 46, 641 (1977)

28. V.P. Goncalves, P. Hepp, Phys. Rev. D 83 (2011)

29. V.P. Gonçalves, D.R. Gratieri, Phys. Rev. D 88(1) (2013)

30. V.P. Goncalves, D.R. Gratieri, Phys. Rev. D 92(11) (2015)

31. J.L. Albacete, J.I. Illana, A. Soto-Ontoso, Phys. Rev. D 92(1) (2015)

32. C.A. Argüelles, F. Halzen, L. Wille, M. Kroll, M.H. Reno, Phys. Rev. D 92(7) (2015)

33. E.L. Berger, M.M. Block, D.W. McKay, C.I. Tan, Phys. Rev. D 77 (2008)

34. M.M. Block, P. Ha, D.W. McKay, Phys. Rev. D 82 (2010)

35. M.M. Block, L. Durand, P. Ha, D.W. McKay, Phys. Rev. D 88(1) (2013)

36. M.M. Block, L. Durand, P. Ha, D.W. McKay, Phys. Rev. D 88(1) (2013)

37. M.G. Aartsen et al. [IceCube Gen2], arXiv:2008.04323 [astroph.HE]

38. R. Devenish, A. Cooper-Sarkar, Deep Inelastic Scattering (University Press, Oxford, 2004)

39. N.N. Nikolaev, B.G. Zakharov, Z. Phys. C 64, 631 (1994)

40. I. Balitsky, Nucl. Phys. B 463, 99-160 (1996)
41. Y.V. Kovchegov, Phys. Rev. D 60 (1999)

42. Y.V. Kovchegov, Phys. Rev. D 61 (2000)

43. E. Iancu, K. Itakura, S. Munier, Phys. Lett. B 590, 199 (2004)

44. G. Soyez, Phys. Lett. B 655, 32 (2007)

45. A.H. Rezaeian, I. Schmidt, Phys. Rev. D 88 (2013)

46. R. Gandhi, C. Quigg, M.H. Reno, I. Sarcevic, Astropart. Phys. 5, 81 (1996)

47. Nancy Wandkowsky for the IceCube Collaboration, TeVPA (2017)

48. M.G. Aartsen et al. [IceCube], arXiv:1710.01191 [astro-ph.HE]

49. S. Dulat et al., Phys. Rev. D 93 (2016)

50. C.Y. Chen, P.S.B. Dev, A. Soni, Phys. Rev. D 89(3), 033012 (2014)

51. L.A. Anchordoqui, H. Goldberg, F. Halzen, T.J. Weiler, Phys. Lett. B 621, 18 (2005)

52. G.L. Fogli, E. Lisi, A. Marrone, D. Montanino, A. Palazzo, Phys. Rev. D 66 (2002)

53. S.S. Wilks, The large-sample distribution of the likelihood ratio for testing composite hypotheses. Ann. Math. Stat. 9(1), 60-62 (1938)

54. M.G. Aartsen et al. [IceCube Collaboration], Astrophys. J. 809(1), $98(2015)$ 